\title{
CRATERS PRODUCED BY EXPLOSIONS ON THE SOIL SURFACE
}

\author{
Daniel Ambrosini*, Bibiana Luccioni ${ }^{\dagger}$, Rodolfo Danesi ${ }^{\dagger}$. \\ * Facultad de Ingeniería, Universidad Nacional de Cuyo \\ Centro Universitario - Parque Gral. San Martín - (5500) Mendoza. Fax 5402614380120 \\ e-mail: dambrosini@uncu.edu.ar, web page: http://fing.uncu.edu.ar/ \\ ${ }^{\dagger}$ Instituto de Estructuras \\ Facultad de Ciencias Exactas y Tecnología, Universidad Nacional de Tucumán \\ Av. Roca 1800, 4000 Tucumán, Argentina. TE: 54-0381-4364087 FAX: 03814364087 \\ e-mail: abest@herrera.unt.edu.ar, web page: http://herrera.unt.edu.ar/iest
}

Keywords: Craters, explosion, blast waves, soils, hydrocode.

\begin{abstract}
In case of terrorist attacks or other intentional actions using explosives, it is extremely important the information that can be obtained from the crater generated by the blast waves. For example, the focus of the explosion and the mass of the explosive used in the attack can be deduced examining the location and dimensions of the crater. However, studies about craters produced by explosions on or above ground level, which would be the case when the explosive charge is situated in a vehicle, are rarely found in the open technical literature. In this paper, a numerical study related to crater produced by explosive loads located on the soil surface is presented. The soil parameters used in the numerical model as well as the analysis procedure were validated against experimental observations of the crater diameters. Results of numerical tests performed with different amounts of explosive on the soil surface are presented. Moreover, the effect of elevation of the center of energy release of explosive loads located on the soil surface is analyzed and discussed. Simple prediction equations for the crater diameter are presented.
\end{abstract}




\section{INTRODUCTION}

Blasting loads have come into attention in recent years due to the great number of accidental or intentional events that affected important structures all over the world, clearly indicating that the issue is relevant for purposes of structural design and reliability analysis. In consequence, extensive research activities in the field of blast loads have taken place in the last few decades.

Dynamic loads due to explosions result in strain rates of the order of $10^{-1}$ to $10^{3} \mathrm{~s}^{-1}$ which imply short time dynamic behaviour of the materials involved, characterised mainly by a great overstrength and increased stiffness, in comparison with normal, static properties. In the case of soils, the response and the mechanism of crater formation are still more complex due to the usual anisotropy and non linear nature of the material, to the variability of mechanical properties and the coexistence of the three phases: solid, liquid and gaseous. Generally, simplifying assumptions must be made in order to solve specific problems. Until now, most practical problems have been solved through empirical approaches. Years of industrial and military experience have been condensed in charts or equations ${ }^{1,2}$. These are useful tools, for example, to establish the weight of explosive to yield a perforation of certain dimensions or to estimate the type and amount of explosive used in a terrorist attack, from the damage registered. Most research is related to underground explosions and only a few papers are concerned with explosions at ground level. Studies about craters produced by explosions above ground level, which would be the case when the explosive charge is situated in a vehicle, are rarely found in the open technical literature. Some reports are classified information limited to government agencies.

On the other hand, most of the information about explosively formed craters found in the literature is based on experimental. Numerical studies were very scarce until some years later.

However, with the rapid development of computer hardware over the last decades, it has become possible to make detailed numerical simulations of explosive events in personal computers, significantly increasing the availability of these methods. New developments in integrated computer hydrocodes complete the tools necessary to carry out the numerical analysis successfully. Nevertheless, it is important to be aware that both these models and analysis procedures still need experimental validation.

A numerical study related to craters produced by explosive charges located on the soil surface is presented in this paper. The analysis is performed with an hydrocode and material models and analysis procedures are validated with experimental results. Additionally, the crater diameters for explosive charges up to $500 \mathrm{~kg}$ of TNT situated on the ground and just at the ground level are obtained. The effect of elevation of the center of energy release of explosive loads located on the soil surface is analyzed and discussed. All the results are compared with empirical equations used nowadays for the prediction of crater dimensions and new simple equations are proposed. 


\section{THEORY AND PREVIOUS RESULTS}

\subsection{Blast waves}

When a condensed high explosive is detonated, a blast wave is formed. A typical pressuretime profile for a blast wave in free air is shown in Figure 1. It is characterized by an abrupt pressure increase at the shock front, followed by a quasi exponential decay back to ambient pressure $p_{0}$ and a negative phase in which the pressure is less than ambient. Of particular importance are the following wave front parameters:

$p_{s}$ : peak overpressure

$T_{s}$ : duration of the positive phase

$i_{s}$ : specific impulse of the wave which is the area beneath the pressure-time curve from the arrival at time $t_{0}$ to the end of the positive phase.

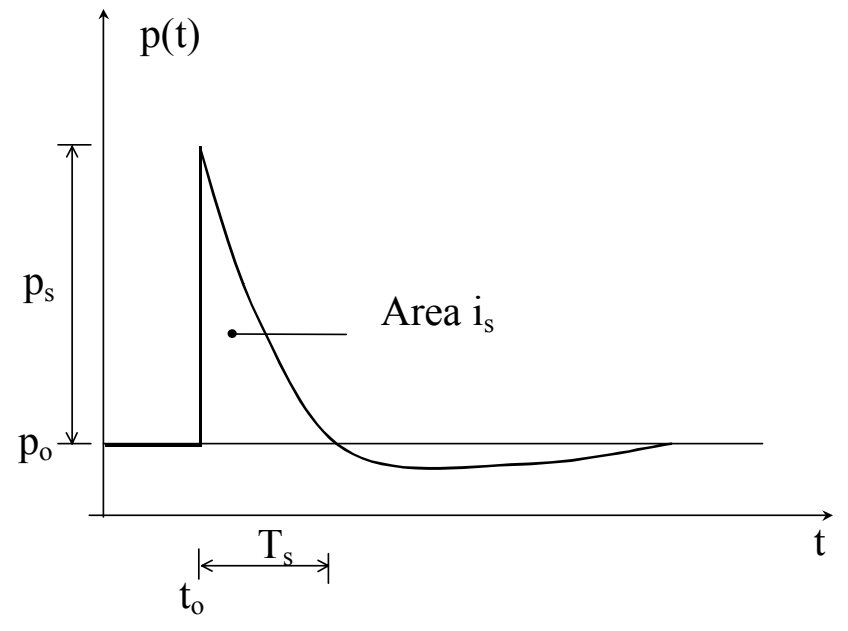

Figure 1. Blast wave pressure-time history

The pressure-time history of a blast wave is often described by exponential functions such as Frielander's equation ${ }^{2}$, which has the form

$$
p(t)=p_{s}\left[1-\frac{t}{T_{s}}\right] \exp \left\{-\frac{b t}{T_{s}}\right\}
$$

where $b$ is a positive constant called the waveform parameter that depends on the peak overpressure $p_{s}$. The most widely used approach to blast wave scaling is Hopkinson's law ${ }^{1}$ which establishes that similar explosive waves are produced at identical scaled distances when two different charges of the same explosive and with the same geometry are detonated in the same atmosphere. Thus, any distance $R$ from an explosive charge $W$ can be transformed into a characteristic scaled distance $Z$ 


$$
Z=\frac{R}{W^{1 / 3}}
$$

The use of $Z$ allows a compact and efficient representation of blast wave data for a wide range of situations. In expression (2), $W$ is the charge mass expressed in kilograms of TNT. In order to quantify blast waves from sources other than TNT, the actual mass of the charge must be converted into an equivalent TNT mass. This is achieved by multiplying the mass of explosive by a conversion factor based on the specific energy, the peak overpressure or the impulse delivered (Baker et al. ${ }^{1}$ ).

\subsection{Crater formation}

A crater produced by an explosive charge situated on or above the ground level is schematized in Figure 2. The crater dimensions defined by Kinney and Graham ${ }^{3}$ are used in this paper (Figure 2): $\mathrm{D}$ is the apparent crater diameter, $\mathrm{D}_{\mathrm{r}}$ is the actual crater diameter and $\mathrm{H}_{2}$ is the apparent depth of the crater. The depth of the crater created by an explosion ordinarily is about one quarter its diameter, but this ratio depends on the type of soil involved. The diameter of the crater produced by an explosion also depends on the relative location of the explosive charge to the ground level. Thus explosions above surface may not create any crater at all ${ }^{3}$.

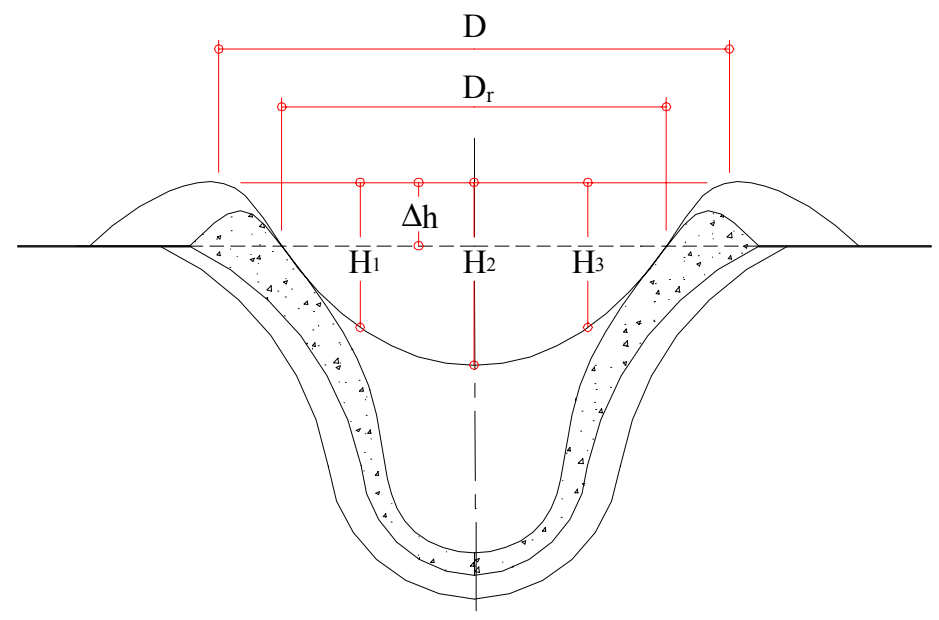

Figure 2. Definitions of the crater dimensions

Tests of crater formation are appropriate tools to study the blast phenomena, the behavior and destructive power of different explosives and the response of soils and rocks under this type of load ${ }^{4}$. The mechanism of crater formation is complex and is related to the dynamic physical properties of air, soil and soil-air interface. Even very carefully performed cratering tests give deviations in the dimensions measured of the order of $10 \%$, while differences of as much as $30 \%$ to $40 \%$ are common (Bull et al. ${ }^{5}$ )

A cavity is always formed when a confined explosion is produced in a mass of soil. If the 
explosion is close to the surface, a crater is formed, a complex interaction taking place between gravity effects, soil strength and transient load conditions. The most important variables in defining the crater shape and size are the mass $W$ of the explosive and the depth of the detonation beneath the air/soil interface $d$. When $d<0$, the explosive is detonated over the air/soil interface, $d=0$ when the detonation occurs in the air/soil interface and $d>0$ when the explosive is detonated beneath the soil surface. For $d>0$, the crater mechanism is altered by gravitational effects. When the depth of the detonation increases, larger amounts of subsoil must be expelled by the explosion. Thus, the crater radius and the depth of the crater increase when $d$ increases, until a certain limit value, from which they rapidly decrease ${ }^{5}$.

Studies concerned with the characteristics of craters caused by explosions usually resort to dimensional analysis and statistics. The scaling law establishes that any linear dimension "L" of the crater can be expressed as a constant multiplied by $W^{\alpha}$ divided by the distance of the charge from de ground, where $W$ represents the equivalent TNT mass of explosive and $\alpha$ is a coefficient depending on if the gravitational effects can be neglected or not. In the first case the cubic root law is applicable $(\alpha=0.33)$ and in the other cases the functional dependence can be quite complex.

Baker et al. ${ }^{6}$ present a dimensional study to model the crater formation phenomenon in the case of underground explosions. Six parameters are chosen to define the problem: the explosive mass $W$, the depth of the explosive charge, $d$, the apparent crater radius $R$, the soil density $\rho$, and two strength parameters to define the soil properties: one with the dimensions of a stress $\sigma$, related to soil strength, and other with the dimensions of a force divided by a cubic length $\left(\mathrm{Nm}^{-3}\right) K$, that takes into account gravitational effects.

After a dimensional analysis and many empirical observations, the following functional relation may be obtained ${ }^{6}$.

$$
\frac{R}{d}=f\left(\frac{W^{7 / 24}}{\sigma^{1 / 6} K^{1 / 8} d}\right)
$$

If $\frac{R}{d}$ (scaled radius of the crater) is plotted as a function of $W^{7 / 24} / d$, it can be seen that this relation is close to experimental results and can be approximately simplified by two straight lines, one with a moderate slope for $W^{7 / 24} / d>0.3$ and one steeper for $W^{7 / 24} / d<0.3$. For $W^{7 / 24} / d<0.3$, the scaled radius of the crater is sensible to small changes in the independent parameter and, due to this fact, the independent parameter or the scaled radius may exhibit great variability. Experimental conditions are better controlled for $W^{7 / 24} / d>0.3$.

It can be deduced that the specific weight $\rho g$ is the best measure for $K$ and that $\rho c^{2}$ is the best measure for $\sigma$, where $c$ is the seismic velocity in the soil. If experimental results for 
different types of soils are plotted in a $\frac{R}{d}$ versus $\frac{W^{7 / 24}}{\rho^{7 / 24} c^{1 / 3} g^{1 / 8} d}$ graph, it may be clearly seen that there is very little variability in the results.

The preceding paragraph refers to underground explosions. There is less information about explosions at ground level. Statistical studies of about 200 accidental above-ground explosions of relative large magnitude are presented by Kinney and Graham ${ }^{3}$. The results exhibit a variation coefficient of about $30 \%$. From these results, the following empirical equation for the crater diameter is proposed.

$$
D[m]=0.8 W[K g]^{1 / 3}
$$

Additional experimental evidence was obtained during the surface explosions performed by EMRTC (Energetic Materials Research Center of the Mineralogical and Technologic Institute of New Mexico). EMRTC conducted experimental determinations to explore alternative ways of controlling the blasting power. In this program, the explosion of $250 \mathrm{~kg}$ of TNT situated at ground level formed a $3.8 \mathrm{~m}$ diameter crater.

In connection with the morphological and structural types of the craters, Jones et $\mathrm{al}^{7}$ present an extensive study of high explosion and planetary impact craters and determine three different basic types: (a) bowl-shaped, (b) flat-floored with central uplift and (c) flat floored with multirings. One of the factors that determine the shape is the height of burst. On the other hand, numerical and independent research results presented by Iturrioz et $\mathrm{al}^{8}$ preliminary confirm the formation of the same shapes of craters. Additionally, Gorodilov et al. ${ }^{9}$ present the results of research about the shape and size of craters generated by explosions of underwater surface charges on sand.

In a previous paper, Ambrosini et al. ${ }^{10}$ presented the results of a series of tests performed with different amounts of explosive at short distances above and below ground level, as well as on the soil surface. These results were used in this paper to calibrate the soil parameters of the numerical model as well as to validate the analysis procedure.

\section{NUMERICAL MODEL}

\subsection{Introduction}

Computer codes normally referred as a "hydrocodes" encompass several different numerical techniques in order to solve a wide variety of non-linear problems in solid, fluid and gas dynamics. The phenomena to be studied with such a program can be characterized as highly time dependent with both geometric non-linearities (e.g. large strains and deformations) and material non-linearities (e.g. plasticity, failure, strain-hardening and softening, multiphase equations of state).

While finite element codes are usually based on the equilibrium condition, the hydrocode utilizes the differential equations governing unsteady material dynamic motion: the local conservation of mass, momentum and energy. In order to obtain a complete solution, in 
addition to appropriate initial and boundary conditions, it is necessary to define a further relation between the flow variables. This can be found from a material model, which relates stress to deformation and internal energy (or temperature). In most cases, the stress tensor may be separated into a uniform hydrostatic pressure (all three normal stresses equal) and a stress deviatoric tensor associated with the resistance of the material to shear distortion.

The relation between the hydrostatic pressure, the local density (or specific volume) and local specific energy (or temperature) is known as an equation of state. Because solids are able to withstand a certain amount of tensile stress, it is necessary to consider extending the equations of state into limited regions of negative values of the pressure (tension). However, because the analytic forms derived for ranges of positive pressure may not be valid for extrapolation into the negative regions special attention should be paid in using some forms of equation of state. The hydrodynamic tensile limit, sometimes referred to as $p_{\min }$, is the minimum pressure to which the material can sustain continuous expansion. If the material pressure drops below this limit in a cell it is assumed that the material will fracture, or in some manner lose its uniform and continuous ability to sustain a tensile pressure. This would then form the lower limit of the analytic equation of state. Regardless of the definition of a value of $p_{\min }$ it may be necessary to provide a different analytic form for negative values of pressure from that used for positive values (but taking care to ensure continuity of function and derivatives at $p=0$ ).

While there are many problems that can be calculated using a hydrodynamic equation of state, there are many applications where material strength effects (i.e. its resistance to shearing forces) cannot be ignored and indeed may even dominate. If the material is a solid and has finite shear strength then, in addition to the calculation of the hydrostatic pressure, it is necessary to define relations between shear stress and strain. The methodology followed in this paper is that first formulated by Wilkins ${ }^{11}$ to extend conventional numerical hydrodynamic codes to include the effects of material strength and resistance to shear distortion.

A relation to define the transition between elastic and plastic strain, both in compression and release, and a relation to define the onset of fracture, are also required. The yield criterion governing the transition from elastic to plastic behavior may involve only constant yield strength, or this strength may itself be a function of the degree of strain (work hardening), the rate of strain and/or the temperature of the material (energy dependency).

Real materials are not able to withstand tensile stresses, which exceed the material's local tensile strength. The computation of the dynamic motion of materials assuming that they always remain continuous, even if the predicted local stresses reach very large negative values, will lead to unphysical solutions. Some model has to be constructed to recognize when tensile limits are reached, to modify the computation to deal with this and to describe the properties of the material after this formulation has been applied.

\subsection{Numerical mesh}

In this paper, an Euler formulation is used to model both air and soil. In the Euler 
processors, a control volume method is used to solve the equations that govern conservation of mass, momentum, and energy. The integral and discrete forms of these equations are expressed in conservation form to obtain accurate, stable solutions. Terms producing changes in conserved variables are divided into two groups: Lagrangian or transport (convective). A two-step numerical procedure is used to solve the finite-difference equations. In the first step, the Lagrange step, the Lagrangian form of the equations are updated or advanced one time interval (time step). In the second step, the Euler step, the updated variables are mapped onto the Euler mesh. Multiple materials are handled through either a volume fraction technique or an interface technique originally developed by Youngs ${ }^{12}$. All variables are cell centered. This allows arbitrary shaped control volumes to be formed more readily at the interface between Euler and Lagrange grids, facilitating the computation of fluid-structure or gas-structure interaction problems.

The use of symmetry conditions allows using a two-dimensional (2D) mesh considering axial symmetry. The number of cells required to produce accurate solutions is greatly reduced when compared with a full 3D model. The mesh used for explosive charges situated on the ground level is shown in Figure 3a. The mesh was filled with different materials: air, TNT and soil as indicated in Figure 3 a with different colors.

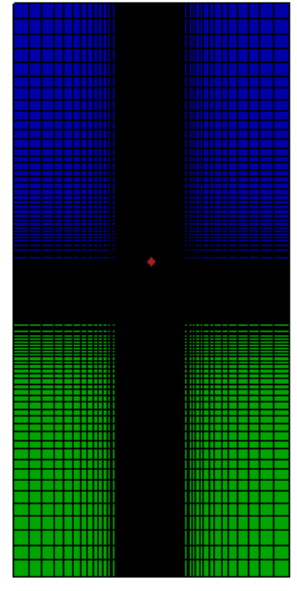

a)

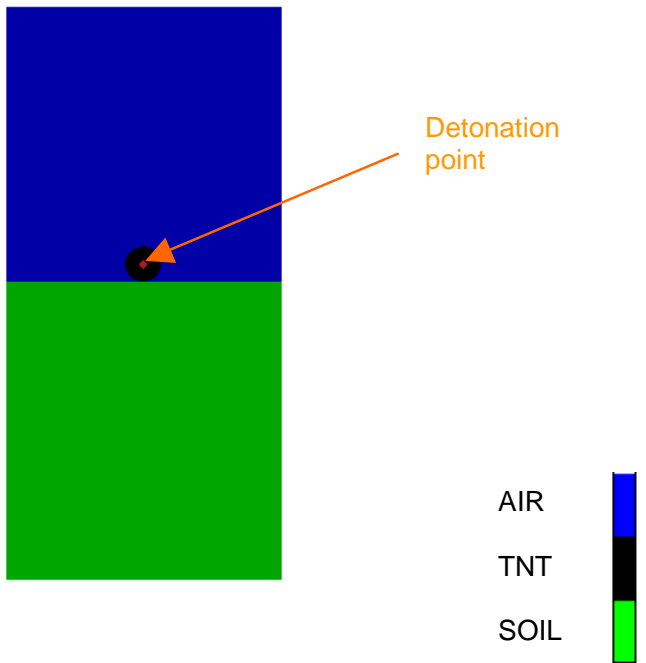

b)

Figure 3. Numerical model for explosives charges situated on the ground level a) Mesh b) Material location

\subsection{Material models}

\subsubsection{Air}

The ideal gas equation of state was used for the air. This is one of the simplest forms of equation of state for gases. In an ideal gas, the internal energy is a function of the temperature alone and if the gas is polytropic the internal energy is simply proportional to temperature. It 
follows that the equation of state for a gas, which has uniform initial conditions, may be written as,

$$
p=(\gamma-1) \rho e
$$

where $p$ is the hydrostatic pressure, $\rho$ is the density and $e$ is the specific internal energy.

$\gamma$ is the adiabatic exponent, it is a constant (equal to $1+R / c_{v}$ ) where constant $\mathrm{R}$ may be taken to be the universal gas constant $R_{0}$ divided by the effective molecular weight of the particular gas and $c_{v}$ is the specific heat at constant volume.

The values of the constants used for air are presented in Table 1.

Table 1: Material properties for Air

\begin{tabular}{|l|}
\hline \multicolumn{1}{|c|}{ EOS: Ideal gas } \\
$\qquad \gamma=1.4$ \\
Reference density $\rho_{o}=1.225 E-3 \mathrm{~g} / \mathrm{cm}^{3}$ \\
Reference temperature: $T_{o}=288.2 \mathrm{~K}$ \\
Specific heat $c_{v}=717.3 \mathrm{~J} / \mathrm{kgK}$ \\
\hline
\end{tabular}

\subsubsection{TNT}

High explosives are chemical substances which, when subject to suitable stimuli, react chemically very rapidly (of order microseconds) releasing energy. In the hydrodynamic theory of detonation, this very rapid time interval is shrunk to zero and a detonation wave is assumed to be a discontinuity which propagates through the unreacted material instantaneously liberating energy and transforming the explosive into detonating products. The normal Rankine-Hugoniot relations, expressing the conservation of mass, momentum and energy across the discontinuity may be used to relate the hydrodynamic variables across the reaction zone. The only difference between the Rankine-Hugoniot equations for a shock wave in a chemically inert material and those for a detonation wave is the inclusion of a chemical energy term in the energy conservation equation.

Since the 1939-45 war, when there was naturally extensive study of the behavior of high explosives, there has been a continuous attempt to understand the detonation process and the performance of the detonation products, leading to considerable improvements in the equation of state of the products. The most comprehensive form of equation of state developed over this period, the"Jones - Wilkins - Lee" (JWL) equation of state is used in this paper,

$$
p=C_{1}\left(1-\frac{\omega}{r_{1} v}\right) e^{-r_{1} v}+C_{2}\left(1-\frac{\omega}{r_{2} v}\right) e^{-r_{2} v}+\frac{\omega e}{v}
$$

Where $v=1 / \rho$ is the specific volume, $\mathrm{C} 1, \mathrm{r} 1, \mathrm{C} 2, \mathrm{r} 2$ and $\omega$ (adiabatic constant) are constants and their values have been determined from dynamic experiments and are available 
in the literature for many common explosives. The values used for TNT are presented en Table 2.

It can be shown that at large expansion ratios the first and second terms on the right hand side of Equation (6) become negligible and hence the behavior of the explosive tends towards that of an ideal gas. Therefore, at large expansion ratios, where the explosive has expanded by a factor of approximately 10 from its original volume, it is valid to switch the equation of state for a high explosive from JWL to ideal gas. In such a case the adiabatic exponent for the ideal gas, $\gamma$, is related to the adiabatic constant of the explosive, $\omega$, by the relation $\gamma=\omega+1$. The reference density for the explosive can then be modified and the material compression will be reset. Potential numerical difficulties are therefore avoided.

An explosive may be initiated by various methods of delivering energy to it. However whether an explosive is dropped, thermally irradiated or shocked, either mechanically or from a shock from an initiator (of more sensitive explosive), initiation of an explosive always goes through a stage in which a shock wave is an important feature. Lee-Tarver equation of state ${ }^{13}$ was used to model both the detonation and expansion of TNT in conjunction with JWL EOS to model the unreacted explosive.

Table 2: Material properties for TNT

\begin{tabular}{|l|}
\hline \multicolumn{1}{|c|}{ EOS: JWL } \\
\hline Reference density $\rho_{o}=1.63 \mathrm{~g} / \mathrm{cm}^{3}$ \\
$\mathrm{C}_{1}=3.7377 \mathrm{E} 8 \mathrm{kPa}$ \\
$\mathrm{C}_{2}=3.73471 \mathrm{E} 6 \mathrm{kPa}$ \\
$\mathrm{r}_{1}=4.15$ \\
$\mathrm{r}_{2}=0.9$ \\
$\omega=0.35$ \\
C-J detonation velocity: $6.93 \mathrm{E} 3 \mathrm{~m} / \mathrm{s}$ \\
C-J energy $/$ unit volumen: $6 \mathrm{E} 6 \mathrm{KJ} / \mathrm{m}^{3}$ \\
C-J pressure: $2.1 \mathrm{E} 7 \mathrm{kPa}$
\end{tabular}

\subsubsection{Soil}

A shock equation of state combined with an elastoplastic strength model based on Mohr Coulomb criterion and an hydro tensile limit were used for the soil.

A Mie-Gruneisen form of equation of state based on the shock Hugoniot was used. The Rankine-Hugoniot equations for the shock jump conditions can be regarded as defining a relation between any pair of the variables $\rho, p, e, u_{p}$ (material velocity behind the shock ) and $U$ (shock velocity). In many dynamic experiments making measurements of $u_{p}$ and $U$ it has been found that for most solids and many liquids over a wide range of pressure there is an empirical linear relationship between these two variables 


$$
U=c_{o}+s u_{p}
$$

This is the case even up to shock velocities around twice the initial sound speed $c_{o}$ and shock pressures of order $100 \mathrm{GPa}$. In this case the equation of state results:

$$
p=p_{H}+\Gamma \rho\left(e-e_{H}\right) \text { with } \quad p_{H}=\frac{\rho_{o} c_{o}^{2} \mu(1+\mu)}{[1-(s-1) \mu]^{2}} ; e_{H}=\frac{1}{2} \frac{p_{H}}{\rho_{o}} \frac{\mu}{1+\mu} ; \quad \mu=\frac{\rho}{\rho_{o}}-1
$$

where $\Gamma$ is the Gruneisen Gamma parameter and it is assumed that $\Gamma \rho=\Gamma_{\mathrm{o}} \rho_{o}=$ const

An elastoplastic model with Mohr Coulomb yield criterion was used for the strength effects. This model is an attempt to reproduce the behavior of dry soil where the cohesion and compaction result in an increasing resistance to shear up to a limiting value of yield strength as the loading increases. This is modeled by a piecewise linear variation of yield stress with pressure. In tension (negative values of $\mathrm{p}$ ) soils have little tensile strength and this is modeled by dropping the curve for $\mathrm{Y}(\mathrm{p})$ rapidly to zero as $\mathrm{p}$ goes negative to give a realistic value for the limiting tensile strength.

A non associated flow rule (Prandtl-Reuss type ) that avoids the problem of shear induced dilatancy in soils was used.

A constant hydrodynamic tensile limit was specified as failure criterion.

All the material properties used for the soil are presented in Table 3.

Table 3: Material properties for soil

\begin{tabular}{|l|}
\hline \multicolumn{1}{|c|}{ EOS: Shock $\quad$ Strength: Mohr Coulomb } \\
\hline Reference density $\rho_{o}=1.92 \mathrm{~g} / \mathrm{cm}^{3}$ \\
Gruneisen Gamma $\Gamma=0.11$ \\
$\mathrm{c}_{0}=1.614 \mathrm{E} 3 \mathrm{~m} / \mathrm{s}$ \\
$\mathrm{s}=1.5$ \\
Shear Modulus G=2.18E5 $\mathrm{kPa}$ \\
Pressure $1=0 \mathrm{kPa} \quad$ Yield stress $1=6.2 \mathrm{E} 3 \mathrm{kPa}$ \\
Pressure $2=1.1 \mathrm{E} 4 \mathrm{Kpa} \quad$ Yield stress $2=6.2 \mathrm{E} 3 \mathrm{kPa}$ \\
Pressure $3=1.0 \mathrm{E} 8 \mathrm{kPa} \quad$ Yield stress $3=6.2 \mathrm{E} 3 \mathrm{kPa}$ \\
Hydro tensile limit $\mathrm{p}_{\min }=-100 \mathrm{kPa}$ \\
\hline
\end{tabular}

\section{RESULTS AND DISCUSSION}

The process of crater formation and crater dimension for explosive charges up to $500 \mathrm{~kg}$ of TNT situated on the ground level were analyzed with the procedure described. 


\subsection{Crater formation}

The process of crater formation for an spherical explosive load of $400 \mathrm{~kg}$ of TNT lying on the ground is illustrated in Figure 4. It may be observed that it takes about $10 \mathrm{~ms}$ to the hole crater to be formed.

\subsection{Comparison with experimental results}

In order to validate not only the material models and material properties but also the analysis procedures a comparison with experimental results was first performed. The results of a series of tests performed with different amounts of explosive from $1 \mathrm{~kg}$ to $10 \mathrm{~kg}$ of TNT on the soil surface ${ }^{10}$ were used to calibrate the materials parameters. Additional comparison was made with. EMRTC experimental determinations. Numerical results and the comparison with experimental ones for both programs are presented in Table 4.

It may be observed that a mean difference of $6.4 \%$ is obtained with respect to experimental results of charges between 1 to $10 \mathrm{~kg}$ of TNT and only $1 \%$ of difference with the experimental result for a greater charge.

a)

b)

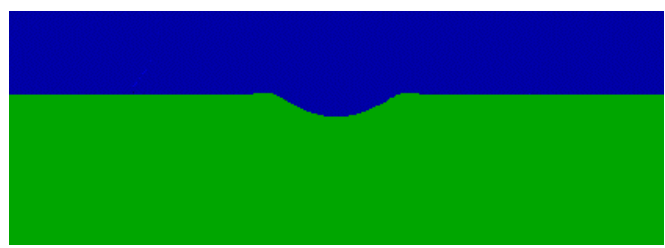

c)

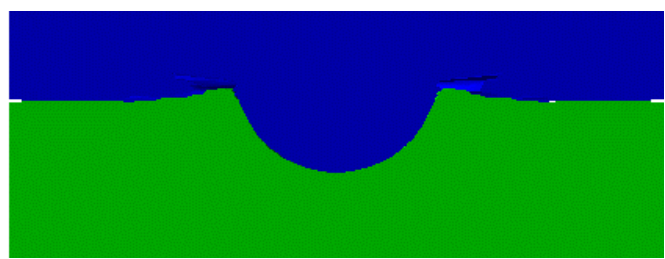

e)

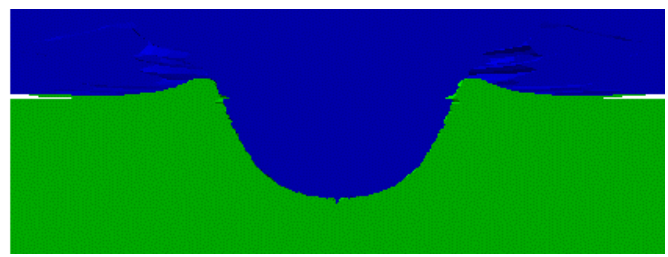

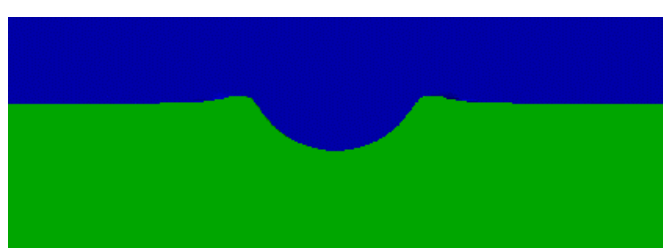

d)

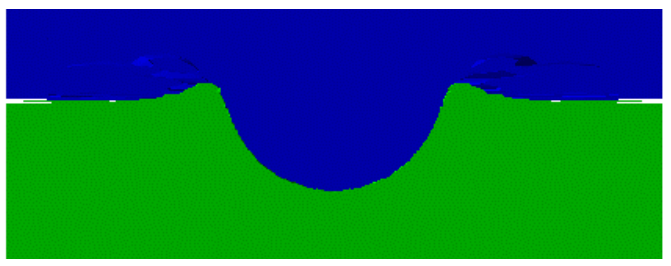

f)

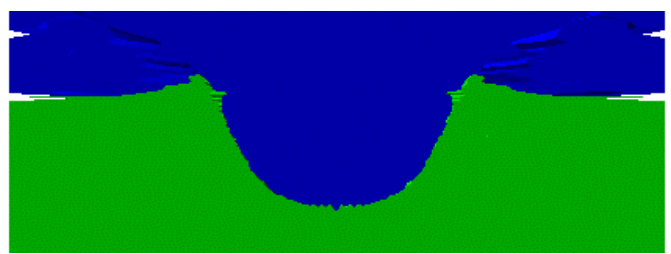

Figure 4: Crater formation (400 kg TNT on the ground)

a) $\mathrm{t}=0.46 \mathrm{~ms}$; b) $\mathrm{t}=1.33 \mathrm{~ms}$; c) $\mathrm{t}=2.77 \mathrm{~ms}$; d) $\mathrm{t}=4.5 \mathrm{~ms}$; e) $\mathrm{t}=6.43 \mathrm{~ms}$; f) $\mathrm{t}=8.04 \mathrm{~ms}$ 
Table 4: Apparent crater diameter. Comparison with experimental results

\begin{tabular}{|c|c|c|c|c|}
\hline Experimental program & $\mathrm{W}[\mathrm{kg}$ of $\mathrm{TNT}]$ & $\begin{array}{ll}\text { Exper. } & \text { results }^{10} \\
D[\mathrm{~m}] & \\
\end{array}$ & Numer. results $\mathrm{D}[\mathrm{m}]$ & Numer/Experim. \\
\hline \multirow[t]{5}{*}{ Ref. 10} & 1 & 0.58 & 0.56 & 0.97 \\
\hline & 2 & 0.74 & 0.68 & 0.92 \\
\hline & 4 & 0.84 & 0.96 & 1.14 \\
\hline & 7 & 1.48 & 1.02 & 0.69 \\
\hline & 10 & 1.56 & 1.50 & 0.96 \\
\hline EMRTC $\bigcirc$ Explosive & 250 & 3.80 & 3.78 & 0.99 \\
\hline
\end{tabular}

\subsection{Numerical results.}

The crater dimensions for explosive charges from 50 to $500 \mathrm{Kg}$ of TNT situated on the ground and with the energy release center at the ground level are calculated in this section. This charges were used because they are in the medium range of terrorist attacks to buildings. The range of explosive masses used in terrorist attacks is discussed in some papers ${ }^{14,15}$ and is strongly dependent on the how the explosive is supposed to have been transported. In order to carry out a comparable analysis, the mass of the explosive is defined by TNT masses. The corresponding masses for other explosives can be obtained through the concept of TNT equivalence $^{16}$. The results obtained are presented in Table 5.

Table 5: Apparent crater diameter

\begin{tabular}{|c|c|c|c|}
\hline W [kg of TNT] & a) $\bigcirc \mathrm{D}[\mathrm{m}]$ & $\begin{array}{l}\text { Comparison } \\
\mathrm{D}_{(\mathrm{a})} \mathrm{D}_{(\mathrm{b})}\end{array}$ \\
\hline \hline 50 & 1.94 & 2.76 & 0.70 \\
\hline 100 & 2.10 & 2.92 & 0.72 \\
\hline 150 & 2.30 & 3.40 & 0.67 \\
\hline 200 & 2.30 & 3.58 & 0.64 \\
\hline 250 & 2.42 & 3.78 & 0.64 \\
\hline 300 & 2.76 & 3.94 & 0.70 \\
\hline 400 & 3.06 & 4.50 & 0.68 \\
\hline 500 & 3.16 & 4.60 & 0.69 \\
\hline
\end{tabular}

It may be observed that the crater is always smaller when the explosive is lying on the ground level than when the energy release center is at the ground level. The difference is attributed to the fact that the energy release center is elevated from the ground level in case (a). Nevertheless, the ratio between apparent crater diameters of both cases is almost constant, about 0.68 , for all the charges studied.

The results of this numerical analysis are also plotted in Figure 5 to be compared with experimental ones and empirical equation (4). 


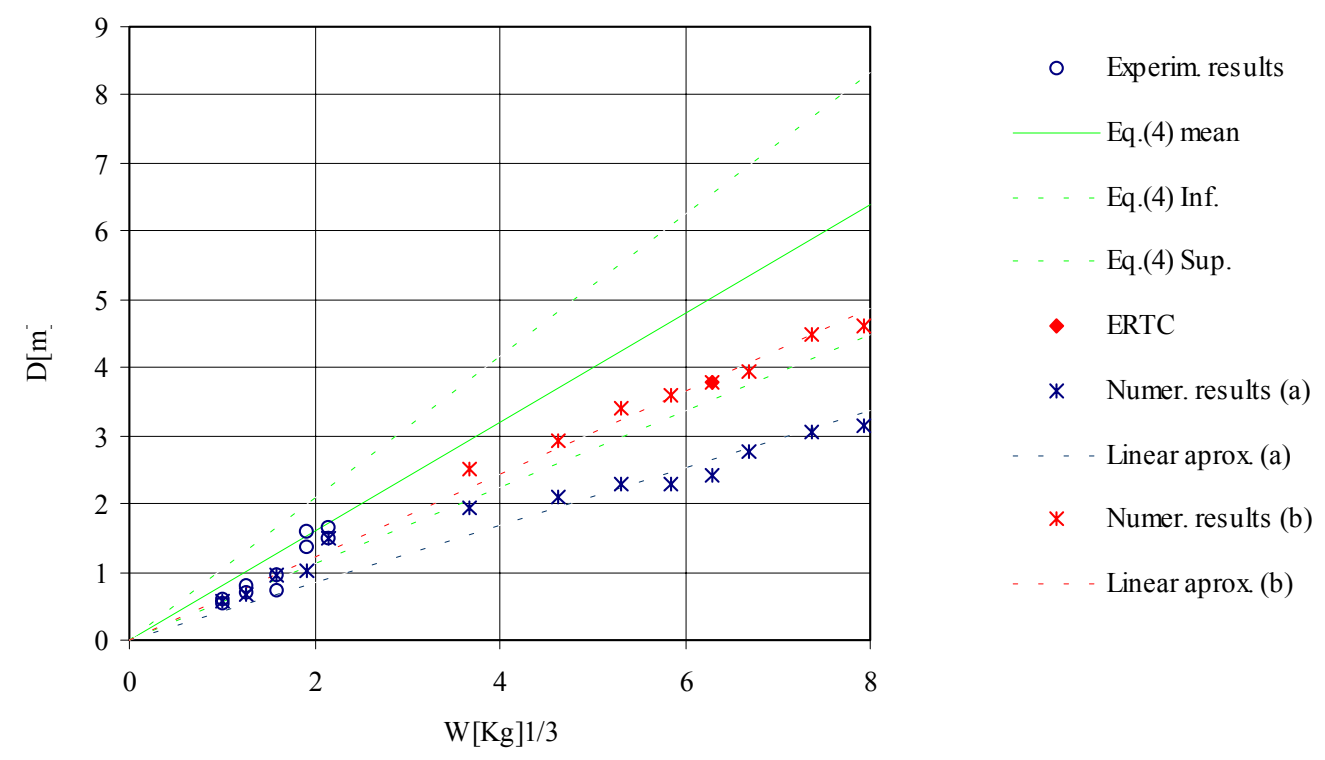

Figure 5. Apparent crater diameter for explosions at the ground level

In a graphic representing crater diameter as a function of the cubic root of the explosive mass, numerical results can be approximatelty represented with two straight lines through the origin. These lines are similar to that describe in equation (4) that was also included in Figure 5 together with its upper and lower limits. Numerical results (b) for explosions with the energy release center at the ground level and EMRTC experimental result are very close to the lower limit of Equation (4). Another linear aproximation must be used for explosives lying on the ground as those simulated in numerical serie (a).

From the previous analysis, Equations (9) and (10) can be proposed for the prediction of crater dimensions in cases (a) and (b) respectively. These equations represent the linear approximation of numerical result by minimum squares.

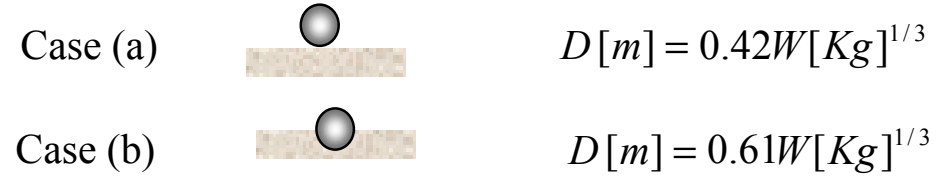

\section{CONCLUSIONS}

A numerical study of crater produced by explosive loads was presented in this paper. Materials models and analysis procedures were validated with experimental results. A good agreement was found with existent results about apparent diameters of this type of craters.

The crater diameters for explosive loads from $50 \mathrm{Kg}$ to $500 \mathrm{~kg}$ of TNT on the soil surface and with the energy release center at the ground level were obtained. The results obtained confirm that simple empirical linear laws that are proposed in the paper can be used to predict 
the apparent crater diameter as a function of the cubic root of the explosive mass. Moreover, the effect of the elevation of the energy release center when the explosive is on the ground is clearly evidenced in numerical results and in the empirical relation proposed.

\section{REFERENCES}

[1]. Baker W.E., Cox P.A., Westine P.S., Kulesz J.J., Strehlow R.A., Explosion hazards and evaluation. Elsevier, Amsterdam (1983).

[2]. Smith PD, Hetherington JG, Blast and Ballistic Loading of Structures, ButterworthHeinemann Ltd, Great Britain. (1994).

[3]. Kinney G.F., Graham K.J. Explosive shocks in air. 2nd Edition, Springer Verlag, (1985).

[4]. Persson PA, Holmberg R., Lee J, Rock blasting and explosives engineering, CRC Press, USA, 540 ps (1994).

[5]. Bull JW, Woodford CH, "Camouflets and their effects on runway supports". Computer and Structures, 69/6, pp 695-706 (1998).

[6]. Baker WE, Westine PS, Dodge FT, Similarity methods in engineering dynamics. Elsevier, Amsterdam (1991).

[7]. Jones GHS, Roddy DJ, Henny RW, Slater JE, “Defence Research Establishment Suffield Explosion Craters and Planetary Impact Craters: Morphological and Structural Deformation Analogues", $15^{\text {th }}$ International Symposium on Military Aspect of Blast and Shock, Alberta, Canada (1997).

[8]. Iturrioz I, Riera JD, "Numerical Study of the Effect of Explosives on a Plane Surface", XII Congress on Numerical Methods and their Applications, ENIEF 2001, Arg. (2001).

[9]. Gorodilov LV, Sukhotin AP, "Experimental investigation of craters generated by explosions of underwater surface charges on sand". Combustion, Explosion, and Shock Waves, Vol. 32, No 3, pp 344-346 (1996).

[10]. Ambrosini, R.D., Luccioni B.M., Danesi R.F., Riera J.D. and Rocha M.M. "Size of Craters Produced by Explosive Charges on or Above the Ground Surface". Shock Waves, Springer Verlag. 12(1), 69-78, (2002).

[11] Wilkins, M. L. "Calculation of Elastic-Plastic Flow", Methods of Computational Physics, 3, 211-263 (1964).

[12]Youngs, D. L. "Time-Dependent Multimaterial Flow with Large Fluid Distortion.", Numerical Methods for Fluid Dynamics, (1982).

[13] Lee, E. L., \& Tarver, C. M.. "Phenomenological Model of Shock Initiation in Heterogeneous Explosives." Physics of Fluids, 23 (12), 2362-2372 (1980).

[14] Elliot C.L., Mays G.C. and Smith P.D. "The protection of buildings against terrorism and disorder”. Proceedings of Ins. of Civil Engs: Structures \& Buildings, 94, 287-297 (1992).

[15] Millington, G. "Discussion of 'The protection of buildings against terrorism and disorder' Proc. of Inst. of Civil Engineers: Structures \& Buildings, 104, 343-350 (1994).

[16]. Formby S.A., Wharton R.K. "Blast Characteristics and TNT Equivalence Values for Some Commercial Explosives Detonated at Ground Level". Journal of Hazardous Materials, 50, 183-198 (1996). 